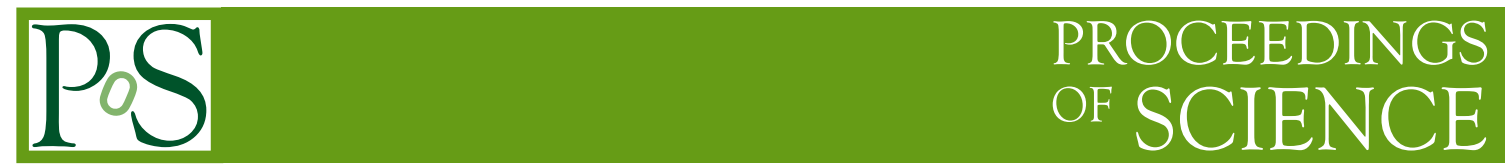

\title{
Gravitational models with non-local scalar fields
}

\section{Sergey Yu. Vernov*}

Skobeltsyn Institute of Nuclear Physics, Moscow State University, Leninskie Gory, 119991, Moscow, Russia

E-mail: svernov@theory.sinp.msu.ru

A general class of $f(R)$ gravity models with minimally coupling a nonlocal scalar field is considered. The Ostrogradski representation for nonlocal gravitational models with a quadratic potential and the way of its localization are proposed. We study the action with an arbitrary analytic function $\mathscr{F}\left(\square_{g}\right)$, which has both simple and double roots. The way of localization allows to find particular solutions of nonlocal equations of gravity.

The XIXth International Workshop on High Energy Physics and Quantum Field Theory, QFTHEP2010 September 08-15, 2010

Golitsyno, Moscow, Russia

\footnotetext{
* Speaker.
} 


\section{Introduction}

Recently a new class of cosmological models based on the string field theory (SFT) [1] and the $p$-adic string theory emerges and attracts a lot of attention [2]-[20]. It is known that the SFT and the $p$-adic string theory are UV-complete ones. Thus, one can expect that resulting (effective) models should be free of pathologies. These models exhibit one general non-standard property, namely, their actions have terms with infinitely many derivatives, i.e. nonlocal terms. The higher derivative terms usually produce phantom fields [21, 22] (see also [23]). Models that includes phantoms violate the null energy condition (NEC), and, therefore, are unstable. Models with higher derivative terms produce also well-known problems with quantum instability [23].

To obtain a stable model with the NEC violation (the state parameter $w_{\mathrm{DE}}<-1$ ) one should construct this model as an effective model, connected with the fundamental theory, which is stable and admits quantization. With the lack of quantum gravity, we can just trust string theory or deal with an effective theory admitting the UV completion.

The purpose of this paper is to study $f(R)$ gravity models with a nonlocal scalar field. We consider a general form of nonlocal action for the scalar field with a quadratic potential, keeping the main ingredient, the analytic function $\mathscr{F}\left(\square_{g}\right)$, which in fact produces the nonlocality, almost unrestricted.

\section{Nonlocal gravitation models}

The SFT inspired nonlocal gravitation models [2] are introduced as a sum of the SFT action of the tachyon field $\phi$ plus the gravity part of the action. One cannot deduce this form of the action from the SFT. In this paper we study the $f(R)$ gravity, which is a straightforward modification of the general relativity. We consider the following action:

$$
S_{f}=\int d^{4} x \sqrt{-g}\left(\frac{f\left(L^{2} R\right)}{16 \pi G_{N} L^{2}}+\frac{1}{\alpha^{\prime} g_{o}^{2}}\left(\frac{1}{2} \phi \mathscr{F}\left(\alpha^{\prime} \square_{g}\right) \phi-V(\phi)\right)-\Lambda\right),
$$

where $f\left(L^{2} R\right)$ is an arbitrary differentiable function. We use the signature $(-,+,+,+), g_{\mu \nu}$ is the metric tensor, $G_{N}$ is the Newtonian constant. The potential $V(\phi)$ is a quadratic polynomial $V(\phi)=C_{2} \phi^{2}+C_{1} \phi+C_{0}$, where $C_{2}, C_{1}$ and $C_{0}$ are arbitrary real constants.

The function $\mathscr{F}$ is assumed to be analytic at all finite points of the complex plane, in other words, to be an entire function. The function $\mathscr{F}$ can be represented by the convergent series expansion: $\mathscr{F}\left(\square_{g}\right)=\sum_{n=0}^{\infty} f_{n} \square_{g}^{n}$. The Weierstrass factorization theorem asserts that the function $\mathscr{F}$ can be represented by a product involving its zeroes $J_{k}$ :

$$
\mathscr{F}(J)=J^{m} e^{Y(J)} \prod_{k=1}^{\infty}\left(1-\frac{J}{J_{k}}\right) e^{\frac{J}{J_{k}}+\frac{J^{2}}{2 \nu_{k}}+\ldots+\frac{1}{p_{k}}\left(\frac{J}{J_{k}}\right)^{p_{k}}},
$$

where $m$ is an order of the root $J=0$ ( $m$ can be equal to zero), $Y(J)$ is an entire function, natural numbers $p_{n}$ are chosen such that the series $\sum_{n=1}^{\infty}\left(\frac{J}{J_{n}}\right)^{p_{n}+1}$ is an absolutely and uniformly convergent one.

Scalar fields $\phi$ (associated with the open string tachyon) is dimensionless, while $\left[\alpha^{\prime}\right]=$ length ${ }^{2}$, $[L]=$ length and $\left[g_{o}\right]=$ length. Let us introduce dimensionless coordinates $\bar{x}_{\mu}=x_{\mu} / \sqrt{\alpha^{\prime}}$, the 
dimensionless Newtonian constant $\bar{G}_{N}=G_{N} / \alpha^{\prime}$, the dimensionless parameter $\bar{L}=L / \sqrt{\alpha^{\prime}}$, and the dimensionless open string coupling constant $\bar{g}_{o}=g_{o} / \sqrt{\alpha^{\prime}}$. The dimensionless cosmological constant $\bar{\Lambda}=\Lambda \alpha^{\prime 2}, \bar{R}$ is the curvature scalar in the coordinates $\bar{x}_{\mu}$ :

$$
S_{f}=\int d^{4} \bar{x} \sqrt{-g}\left(\frac{f\left(\bar{L}^{2} \bar{R}\right)}{16 \pi \bar{G}_{N} \bar{L}^{2}}+\frac{1}{\bar{g}_{o}^{2}}\left(\frac{1}{2} \phi \mathscr{F}\left(\bar{\square}_{g}\right) \phi-V(\phi)\right)-\bar{\Lambda}\right),
$$

In the following formulae we omit bars, but use only dimensionless coordinates and parameters.

It is well-known [24] that at $f^{\prime}(R)>0$ any $f(R)$ gravity models in the metric variational approach are equivalent to the Einstein gravity with a scalar field ${ }^{1}$. In the metric variational approach the equations of gravity are as follows:

$$
G_{\mu v} \equiv f^{\prime}(R) R_{\mu v}-\frac{f(R)}{2} g_{\mu v}-D_{\mu} \partial_{v} f^{\prime}(R)+g_{\mu v} \square_{g} f^{\prime}(R)=8 \pi G_{N} T_{\mu v}, \quad \mathscr{F}\left(\square_{g}\right) \phi=\frac{d V}{d \phi},
$$

where the energy-momentum (stress) tensor $T_{\mu \nu}$ is:

$$
\begin{gathered}
T_{\mu \nu} \equiv-\frac{2}{\sqrt{-g}} \frac{\delta S}{\delta g^{\mu \nu}}=\frac{1}{g_{o}^{2}}\left(E_{\mu v}+E_{v \mu}-g_{\mu \nu}\left(g^{\rho \sigma} E_{\rho \sigma}+W\right)\right), \\
E_{\mu v} \equiv \frac{1}{2} \sum_{n=1}^{\infty} f_{n} \sum_{l=0}^{n-1} \partial_{\mu} \square_{g}^{l} \phi \partial_{v} \square_{g}^{n-1-l} \phi, \quad W \equiv \frac{1}{2} \sum_{n=2}^{\infty} f_{n} \sum_{l=1}^{n-1} \square_{g}^{l} \phi \square_{g}^{n-l} \phi-\frac{f_{0}}{2} \phi^{2}+C_{1} \phi .
\end{gathered}
$$

\section{Localization of nonlocal gravitational actions}

The Ostrogradski representation has been proposed for polynomial $\mathscr{F}(\square)$ in the Minkowski space-time [21, 22]. Our goal to generalize this result on gravitational models with an arbitrary analytic function $\mathscr{F}(\square)$ with simple and double roots. We also generalize the Ostrogradski representation on the models with a linear potential. The nonlocal cosmological models with quadratic potentials have been studied in $[6,7,13,14,15,16,18]$.

Let us start with the case $C_{1}=0$. We consider a function $\mathscr{F}(J)$, which has simple roots $J_{i}$ and double roots $\tilde{J}_{k}$, and the function

$$
\phi_{0}=\sum_{i=1}^{N_{1}} \phi_{i}+\sum_{k=1}^{N_{2}} \tilde{\phi}_{k}
$$

where

$$
\left(\square_{g}-J_{i}\right) \phi_{i}=0 \quad \text { and } \quad\left(\square_{g}-\tilde{J}_{k}\right)^{2} \tilde{\phi}_{k}=0 \quad \Leftrightarrow \quad\left(\square_{g}-\tilde{J}_{k}\right) \tilde{\phi}_{k}=\varphi_{k}, \quad\left(\square_{g}-\tilde{J}_{k}\right) \varphi_{k}=0 .
$$

Without loss of generality we assume that for any $i_{1}$ and $i_{2} \neq i_{1}$ conditions $J_{i_{1}} \neq J_{i_{2}}$ and $\tilde{i_{1_{1}}} \neq \tilde{J_{i_{2}}}$ are satisfied.

The energy-momentum tensor, which corresponds to $\phi_{0}$, has the following form:

$$
T_{\mu v}\left(\phi_{0}\right)=T_{\mu v}\left(\sum_{i=1}^{N_{1}} \phi_{i}+\sum_{k=1}^{N_{2}} \tilde{\phi}_{k}\right)=\sum_{i=1}^{N_{1}} T_{\mu v}\left(\phi_{i}\right)+\sum_{k=1}^{N_{2}} T_{\mu v}\left(\tilde{\phi}_{k}\right),
$$

\footnotetext{
${ }^{1}$ There are two types of $f(R)$ gravity: the metric variational approach and the Palatini formalism. In the first case the equations of motion are obtained by variation with respect to metric. Connections are the function of metric in this formalism. In the Palatini formalism one should vary the action independently with respect to metric and the connections.
} 
where all $T_{\mu \nu}$ are given by (2.5) and

$$
\begin{gathered}
E_{\mu \nu}\left(\phi_{i}\right)=\frac{\mathscr{F}^{\prime}\left(J_{i}\right)}{2} \partial_{\mu} \phi_{i} \partial_{\nu} \phi_{i}, \quad E_{\mu \nu}\left(\tilde{\phi}_{k}\right)=\frac{\mathscr{F}^{\prime \prime}\left(\tilde{J}_{k}\right)}{4}\left(\partial_{\mu} \tilde{\phi}_{k} \partial_{\nu} \varphi_{k}+\partial_{\nu} \tilde{\phi}_{k} \partial_{\mu} \varphi_{k}\right)+\frac{\mathscr{F}^{\prime \prime \prime}\left(\tilde{J}_{k}\right)}{12} \partial_{\mu} \varphi_{k} \partial_{\nu} \varphi_{k}, \\
W\left(\phi_{i}\right)=\frac{J_{i} \mathscr{F}^{\prime}\left(J_{i}\right)}{2} \phi_{i}^{2}, \quad W\left(\tilde{\phi}_{k}\right)=\frac{\tilde{J}_{k} \mathscr{F}^{\prime \prime}\left(\tilde{J}_{k}\right)}{2} \tilde{\phi}_{k} \varphi_{k}+\left(\frac{\tilde{J}_{k} \mathscr{F}^{\prime \prime \prime}\left(\tilde{J}_{k}\right)}{12}+\frac{\mathscr{F}^{\prime \prime}\left(\tilde{J}_{k}\right)}{4}\right) \varphi_{k}^{2},
\end{gathered}
$$

where a prime denotes a derivative with respect to $J: \mathscr{F}^{\prime} \equiv \frac{d \mathscr{F}}{d J}, \mathscr{F}^{\prime \prime} \equiv \frac{d^{2} \mathscr{F}}{d J^{2}}$ and $\mathscr{F}^{\prime \prime \prime} \equiv \frac{d^{3} \mathscr{F}}{d J^{3}}$.

Considering the following local action

$$
S_{\text {loc }}=\int d^{4} x \sqrt{-g}\left(\frac{f(R)}{16 \pi G_{N}}-\Lambda\right)+\sum_{i=1}^{N_{1}} S_{i}+\sum_{k=1}^{N_{2}} \tilde{S}_{k}
$$

where

$$
\begin{gathered}
S_{i}=-\frac{1}{g_{o}^{2}} \int d^{4} x \sqrt{-g} \frac{\mathscr{F}^{\prime}\left(J_{i}\right)}{2}\left(g^{\mu v} \partial_{\mu} \phi_{i} \partial_{v} \phi_{i}+J_{i} \phi_{i}^{2}\right), \\
\tilde{S}_{k}=-\frac{1}{g_{0}^{2}} \int d^{4} x \sqrt{-g}\left(g ^ { \mu v } \left(\frac{\mathscr{F}^{\prime \prime}\left(\tilde{J_{k}}\right)}{4}\left(\partial_{\mu} \tilde{\phi}_{k} \partial_{\nu} \varphi_{k}+\partial_{v} \tilde{\phi}_{k} \partial_{\mu} \varphi_{k}\right)+\right.\right. \\
\left.\left.+\frac{\mathscr{F}^{\prime \prime \prime}\left(\tilde{J_{k}}\right)}{12} \partial_{\mu} \varphi_{k} \partial_{v} \varphi_{k}\right)+\frac{\tilde{J}_{k} \mathscr{F}^{\prime \prime}\left(\tilde{J}_{k}\right)}{2} \tilde{\phi}_{k} \varphi_{k}+\left(\frac{\tilde{J}_{k} \mathscr{F}^{\prime \prime \prime}\left(\tilde{J}_{k}\right)}{12}+\frac{\mathscr{F}^{\prime \prime}\left(\tilde{J}_{k}\right)}{4}\right) \varphi_{k}^{2}\right),
\end{gathered}
$$

we can see that solutions of the Einstein equations and equations in $\phi_{k}, \tilde{\phi}_{k}$ and $\varphi_{k}$, obtained from this action, solves the initial nonlocal equations (2.4). Thus, we obtained that special solutions to nonlocal equations can be found as solutions to system of local (differential) equations. If $\mathscr{F}(J)$ has an infinity number of roots then one nonlocal model corresponds to infinity number of different local models and the initial nonlocal action (2.3) generates infinity number of local actions (3.6).

We should prove that the way of localization is self-consistent. To construct local action (3.6) we assume that equations (3.2) are satisfied. Therefore, the method of localization is correct only if these equations can be obtained from the local action $S_{l o c}$. The straightforward calculations show that the way of localization is self-consistent because:

$$
\frac{\delta S_{l o c}}{\delta \phi_{i}}=0 \Leftrightarrow \square_{g} \phi_{i}=J_{i} \phi_{i} ; \frac{\delta S_{l o c}}{\delta \tilde{\phi}_{k}}=0 \Leftrightarrow \square_{g} \varphi_{k}=\tilde{J}_{k} \varphi_{k} ; \frac{\delta S_{l o c}}{\delta \varphi_{k}}=0 \Leftrightarrow \square_{g} \tilde{\phi}_{k}=\tilde{J}_{k} \tilde{\phi}_{k}+\varphi_{k} .
$$

In spite of the above-mention equations we obtain from $S_{l o c}$ the equations:

$$
G_{\mu v}=8 \pi G_{N}\left(T_{\mu v}\left(\phi_{0}\right)-\Lambda g_{\mu v}\right),
$$

where $\phi_{0}$ is given by (3.1) and $T_{\mu \nu}\left(\phi_{0}\right)$ can be calculated by (3.3). So, we obtained such systems of differential equations that any solutions of these systems are particular solutions of the initial nonlocal equations (2.4).

Let us consider functions $\mathscr{F}(J)$ with two and only two simple roots. If $\mathscr{F}(J)$ has two real simple roots, then $\mathscr{F}^{\prime}(J)>0$ at one root and $\mathscr{F}^{\prime}(J)<0$ at another root, so we get a quintom model [25], in other words, local model with one standard scalar field and one phantom scalar field. In the case of two complex conjugated simple roots $J_{j}$ and $J_{j}^{*}$ one gets the following action:

$$
S_{c}=\int d^{4} x \frac{\sqrt{-g}}{2 g_{o}^{2}}\left(\mathscr{F}^{\prime}\left(J_{j}\right)\left(g^{\mu v} \partial_{\mu} \phi_{j} \partial_{\nu} \phi_{j}+J_{j} \phi_{j}^{2}\right)+\mathscr{F}^{\prime *}\left(J_{j}\right)\left(g^{\mu v} \partial_{\mu} \phi_{j}^{*} \partial_{\nu} \phi_{j}^{*}+J_{j}^{*} \phi_{i}^{* 2}\right)\right) .
$$


We introduce real fields $\xi$ and $\eta$ such that $\phi_{j}=\xi+i \eta, \phi_{j}^{*}=\xi-i \eta$, denote $d_{r} \equiv \Re e\left(\mathscr{F}^{\prime}(J)\right)$, $d_{i} \equiv \mathfrak{I} m\left(\mathscr{F}^{\prime}(J)\right)$, and obtain:

$$
S_{c}=\int d^{4} x \frac{\sqrt{-g}}{2 g_{o}^{2}}\left(d_{r} g^{\mu v}\left(\partial_{\mu} \xi \partial_{v} \xi-\partial_{\mu} \eta \partial_{v} \eta\right)+d_{i} g^{\mu v}\left(\partial_{\mu} \xi \partial_{v} \eta-\partial_{\mu} \eta \partial_{v} \xi\right)+V_{1}\right),
$$

where $V_{1}$ is a potential term. In the case $d_{i}=0$ we get a quintom model, in opposite case the kinetic term in $S_{c}$ has a nondiagonal form. To diagonalize the kinetic term we make the transformation: $\chi=v+\tilde{C} \sigma, \eta=-\tilde{C} v+\sigma$, where $\tilde{C} \equiv\left(d_{r}+\sqrt{d_{r}^{2}+d_{i}^{2}}\right) / d_{i}$, and get a quintom model:

$$
S_{c}=\int d^{4} x \frac{\sqrt{-g}}{2 g_{o}^{2}}\left(\frac{2\left(d_{r}^{2}+d_{i}^{2}\right)}{d_{i}^{2}}\left(d_{r}+\sqrt{d_{r}^{2}+d_{i}^{2}}\right)\left(\partial_{\mu} v \partial_{\nu} v-\partial_{\mu} \sigma \partial_{\nu} \sigma\right)+V_{1}\right) .
$$

In the case of a real double root $\tilde{J}_{k}$ we express $\tilde{\phi}_{k}$ and $\varphi_{k}$ in terms of new fields $\xi_{k}$ and $\chi_{k}$ :

$$
\tilde{\phi}_{k}=\frac{1}{2 \mathscr{F}^{\prime \prime}\left(\tilde{J_{k}}\right)}\left(\left(\mathscr{F}^{\prime \prime}\left(\tilde{J_{k}}\right)-\frac{2}{3} \mathscr{F}^{\prime \prime \prime}\left(\tilde{J_{k}}\right)\right) \xi_{k}-\left(\mathscr{F}^{\prime \prime}\left(\tilde{J_{k}}\right)+\frac{2}{3} \mathscr{F}^{\prime \prime \prime}\left(\tilde{J}_{k}\right)\right) \chi_{k}\right), \quad \varphi_{k}=\xi_{k}+\chi_{k},
$$

we obtain the corresponding $\tilde{S}_{k}$ in the following form:

$$
\begin{aligned}
\tilde{S}_{k} & =\frac{-1}{2 g_{o}^{2}} \int d^{4} x \sqrt{-g}\left(g^{\mu v} \frac{\mathscr{F}^{\prime \prime}\left(\tilde{J_{k}}\right)}{4}\left(\partial_{\mu} \xi_{k} \partial_{\nu} \xi_{k}-\partial_{v} \chi_{k} \partial_{\mu} \chi_{k}\right)+\left[\frac{\tilde{J_{k}} \mathscr{F}^{\prime \prime \prime}\left(\tilde{J_{k}}\right)}{12}+\frac{\mathscr{F}^{\prime \prime}\left(\tilde{J}_{k}\right)}{4}\right]\left(\xi_{k}+\chi_{k}\right)^{2}+\right. \\
& \left.+\frac{\tilde{J_{k}}}{4}\left[\left(\mathscr{F}^{\prime \prime}\left(\tilde{J_{k}}\right)-\frac{2}{3} \mathscr{F}^{\prime \prime \prime}\left(\tilde{J_{k}}\right)\right) \xi_{k}-\left(\mathscr{F}^{\prime \prime}\left(\tilde{J_{k}}\right)+\frac{2}{3} \mathscr{F}^{\prime \prime \prime}\left(\tilde{J_{k}}\right)\right) \chi_{k}\right]\left(\xi_{k}+\chi_{k}\right)\right) .
\end{aligned}
$$

It is easy to see that each $\tilde{S}_{k}$ includes one phantom scalar field and one standard scalar field. So, in the case of one double root we obtain a quintom model. In the Minkowski space appearance of phantom fields in models, when $\mathscr{F}(J)$ has a double root, has been obtained in [22]. So, we come to conclusion that both two simple roots and one double root of $\mathscr{F}(J)$ generate quintom models.

The model with action (2.3) in the case $C_{1} \neq 0$ has been considered in detail in [18]. Here we present only the obtained algorithm of localization for an arbitrary quadratic potential $V(\phi)=$ $C_{2} \phi^{2}+C_{1} \phi+C_{0}$ :

- Change values of $f_{0}$ and $\Lambda$ such that the potential takes the form $V(\phi)=C_{1} \phi$.

- Find roots of the function $\mathscr{F}(J)$ and calculate orders of them. Select an finite number of simple and double roots.

- Construct the corresponding local action. In the case $C_{1}=0$ one should use formula (3.6). In the case $C_{1} \neq 0$ and $f_{0} \neq 0$ one should use (3.6) with the replacement of the scalar field $\phi$ by $\chi$ and the corresponding modification of the cosmological constant. In the case $C_{1} \neq 0$ and $f_{0}=0$ the local action is the sum of (3.6) and either

$$
S_{\psi}=-\frac{1}{2 g_{o}^{2}} \int d^{4} x \sqrt{-g}\left(f_{1} g^{\mu v} \partial_{\mu} \psi \partial_{\nu} \psi+2 C_{1} \psi+\frac{f_{2} C_{1}^{2}}{f_{1}^{2}}\right),
$$

in the case of simple root $J=0$, or

$$
S_{\tilde{\psi}}=-\int d^{4} x \frac{\sqrt{-g}}{2 g_{o}^{2}}\left[g^{\mu \nu}\left(f_{2}\left(\partial_{\mu} \tilde{\psi} \partial_{\nu} \tau+\partial_{\nu} \tilde{\psi} \partial_{\mu} \tau\right)+f_{3} \partial_{\mu} \tau \partial_{\nu} \tau\right)+f_{2} \tau^{2}+2 C_{1} \tilde{\psi}+\frac{f_{3} C_{1}}{2 f_{2}} \tau\right]
$$


in the case of double root $J=0$. Note that in the case $C_{1} \neq 0$ and $f_{0}=0$ the local action (3.6) has no term, which corresponds to the $\operatorname{root} J=0$.

- Vary the obtained local action and get a system of the Einstein equations and equations of motion. The obtained system is a finite order system of differential equations, i.e. we get a local system. Seek solutions of the obtained local system.

\section{Conclusion}

The main result of this paper is the generalization of the algorithm of localization on the $f(R)$ gravity models with a nonlocal scalar field. The algorithm of localization is proposed for an arbitrary analytic function $\mathscr{F}\left(\square_{g}\right)$, which has both simple and double roots. We have proved that the same functions solve the initial nonlocal Einstein equations and the obtained local Einstein equations. We have found the corresponding local actions and proved the self-consistence of our approach. In the case of two simple roots as well as in the case of one double root we get a quintom model [25]. The algorithm of localization does not depend on metric, so it can be used to find solutions in any metrics.

The author wishes to express his thanks to I. Ya. Aref'eva for useful and stimulating discussions. The research has been supported in part by RFBR grant 08-01-00798, grant of Russian Ministry of Education and Science NSh-4142.2010.2 and by Federal Agency for Science and Innovation under state contract 02.740.11.0244.

\section{References}

[1] C.B. Thorn, String field theory, Phys. Rept. 175 (1989) 1-101

K. Ohmori, A Review on Tachyon Condensation in Open String Field Theories, hep-th/ 0102085 I.Ya. Aref'eva, D.M. Belov, A.A. Giryavets, A.S. Koshelev and P.B. Medvedev, Noncommutative Field Theories and (Super)String Field Theories, hep-th/ 0111208 I.Ya. Arefeva, String field theory: From high energy to cosmology, Theor. Math. Phys. 163 (2010) 697-704 [Teor. Mat. Fiz. 163 (2010) 355-365]

[2] I.Ya. Aref'eva, Nonlocal String Tachyon as a Model for Cosmological Dark Energy, AIP Conf. Proc. 826, $p$-Adic Mathematical Physics, eds. A.Yu. Khrennikov, Z. Rakic, I.V. Volovich, AIP, Melville, NY, 2006, pp. 301-311 [astro-ph/0410443]

[3] I.Ya. Aref'eva and L.V. Joukovskaya, Time Lumps in Nonlocal Stringy Models and Cosmological Applications, JHEP 0510 (2005) 087 [hep-th/ 0504200 ]

[4] G. Calcagni, Cosmological tachyon from cubic string field theory, JHEP 0605 (2006) 012, [hep-th/0512259]

[5] N. Barnaby, T. Biswas, and J.M. Cline, p-adic Inflation, JHEP 0704 (2007) 056 [hep-th/0612230]

[6] A.S. Koshelev, Non-local SFT Tachyon and Cosmology, JHEP 0704 (2007) 029 [hep-th/0701103]

[7] I.Ya. Aref'eva, L.V. Joukovskaya, and S.Yu. Vernov, Bouncing and accelerating solutions in nonlocal stringy models JHEP 0707 (2007) 087 [hep-th / 0701184 ] 
[8] I.Ya. Aref'eva and I.V. Volovich, Quantization of the Riemann Zeta-Function and Cosmology, Int. J. of Geom. Meth. Mod. Phys. 4 (2007) 881-895 [hep-th/ 0701284 ]

[9] J.E. Lidsey, Stretching the Inflaton Potential with Kinetic Energy, Phys. Rev. D 76 (2007) 043511 [hep-th/0703007]

[10] G. Calcagni, M. Montobbio, and G. Nardelli, A route to nonlocal cosmology, Phys. Rev. D 76 (2007) 126001 [arXiv:0 0705.3043 ]

G. Calcagni, M. Montobbio, and G. Nardelli, Localization of nonlocal theories, Phys. Lett. B 662 (2008) 285-289 [arXiv:0712.2237]

[11] L.V. Joukovskaya, Dynamics in nonlocal cosmological models derived from string field theory, Phys. Rev. D 76 (2007) 105007 [arXiv: 0707.1545 ]

L.V. Joukovskaya, Dynamics with Infinitely Many Time Derivatives in Friedmann-Robertson-Walker Background and Rolling Tachyon, JHEP 0902 (2009) 045 [arXiv : 0807.2065 ]

[12] N. Barnaby and N. Kamran, Dynamics with Infinitely Many Derivatives: The Initial Value Problem, JHEP 0802 (2008) 008 [arXiv: 0709 . 3968]

N. Barnaby and N. Kamran, Dynamics with Infinitely Many Derivatives: Variable Coefficient Equations, JHEP 0812 (2008) 022 [arXiv : 0809 . 4513]

[13] I.Ya. Aref'eva, L.V. Joukovskaya, and S.Yu. Vernov, Dynamics in nonlocal linear models in the Friedmann-Robertson-Walker metric, J. Phys. A: Math. Theor. 41 (2008) 304003 [arXiv:0711.1364]

[14] D.J. Mulryne and N.J. Nunes, Diffusing non-local inflation: Solving the field equations as an initial value problem, Phys. Rev. D 78 (2008) 063519 [arXiv: 0805.0449 ]

[15] A.S. Koshelev and S.Yu. Vernov, Cosmological perturbations in SFT inspired non-local scalar field models, arXiv:0903.5176; A.S. Koshelev and S.Yu. Vernov, Analysis of scalar perturbations in cosmological models with a non-local scalar field, arXiv:1009.0746

[16] S.Yu. Vernov, Localization of nonlocal cosmological models with quadratic potentials in the case of double roots, Class. Quant. Grav. 27 (2010) 035006 [arXiv: 0907.0468 ]

[17] G. Calcagni, G. Nardelli, Non-local gravity and the diffusion equation, arXiv: 1004.5144

[18] S.Yu. Vernov, Localization of the SFT inspired Nonlocal Linear Models and Exact Solutions, arXiv: 1005.0372

[19] S.Yu. Vernov, Exact Solutions for Nonlocal Nonlinear Field Equations in Cosmology, arXiv: 1005.5007

[20] F. Galli, A.S. Koshelev, Multi-scalar field cosmology from SFT: an exactly solvable approximation, Theor. Math. Phys. (2010) 164, 1169-1175 [Teor. Mat. Fiz. 164 (2010) 401-409], [arXiv:1010.1773]

[21] M. Ostrogradski, Mem. St. Petersbourg VI Series, V. 4 (1850) 385-517

[22] A. Pais and G.E. Uhlenbeck, On Field Theories with Nonlocalized Action, Phys. Rev. 79 (1950) $145-165$

[23] I.Ya. Aref'eva and I.V. Volovich, On the null energy condition and cosmology, Theor. Math. Phys. 155 (2008) 503-511 [Teor. Mat. Fiz. 155 (2008) 3-12], [hep-th / 0612098 ]

[24] V.F. Mukhanov, H.A. Feldman, and R.H. Brandenberger, Theory of Cosmological Perturbations, Phys. Rept. 215 (1992) 203-333

[25] Yi-Fu Cai, E.N. Saridakis, M.R. Setare, and Jun-Qing Xia, Phys. Rep. 493 (2010) 1-60, [arXiv:0909.2776]; Hongsheng Zhang, Crossing the phantom divide, arXiv:0909.3013 